\title{
Síndrome de Lemierre con distrés respiratorio
}

\author{
Lemierre's syndrome with acute respiratory distress syndrome
}

\author{
Miguel Silva, Eduarda Pena, Margarida Oliveira, Daniela Carvalho \\ Department of Emergency and Intensive Medicine, Hospital Pedro Hispano, Unidade de Local de Saúde de Matosinhos, Portugal
}

\begin{abstract}
Lemierre's syndrome is a clinical triad of acute oropharyngeal infection, secondary local invasion and vein thrombosis often involving the internal jugular vein, along with evidence of systemic septic embolism. Usually affecting otherwise healthy adolescents and young adults, anaerobic Fusobacterium spp. are often implicated. Other bacterial species are sometimes found. While being a rare disease with a reported 14.4 cases per million person-years, it carries a mortality rate of 5 to $9 \%$. Clinical presentation can be severe owing to septic shock, multiorgan dysfunction, septic embolization and acute respiratory distress syndrome requiring ICU admission. Treatment involves antibiotic therapy with anaerobic coverage and supportive care. Anticoagulation use is controversial and surgical treatment may be needed to control focus of infection. There is few quality evidence available regarding the clinical management of this syndrome. We report a case of Lemierre syndrome caused by Streptococcus hemolyticus group C, complicated with pulmonary septic embolism, necrotizing pneumonia, empyema and acute respiratory distress syndrome with a degree of severity that required the use of extracorporeal membrane oxygenation to support the patient.
\end{abstract}

Palabras clave: síndrome de Lemierre, Streptococcus, Fusobacterium, síndrome de distrés respiratorio agudo, membrana de oxigenación extracorpórea Keywords: Lemierre syndrome, Streptococcus, Fusobacterium, acute respiratory distress syndrome, extracorporeal membrane oxygenation

\section{INTRODUCTION AND LITERATURE REVIEW}

The exact diagnostic criteria are still debated, but the original description by Lemierre in 1936, who termed it as "anaerobic postanginal septicemia", is quite distinctive, describing not only local thrombophlebitis following an oropharyngeal or neck infection, the isolation of anaerobic species, most commonly Fusobacterium spp. (often combined with other anaerobic species such as anaerobic streptococcus), as well as distant septic embolization, especially to the lungs, where the formation of abscesses were often complicated by pleural effusion and empyema, leading to septic shock and high fatality rate ${ }^{1}$. After the advent of antibiotics in the 40s this entity became progressively rarer, and while in the past few years the frequency of reports has increased in literature $^{3}$, the most current epidemiological study points to an incidence of 14.4 cases per million/year among the 15-24 years of age range, where the highest incidence is found ${ }^{3}$. The mortality rate is reported somewhere between 5 and $9 \%$, however it has fallen from at least $90 \%$ since the introduction of antibiotics 23,4 .

While Fusobacterium spp., particularly Fusobacterium necrophorum, remain the most commonly reported agent ${ }^{4}$, other bacterial species, mostly anaerobes, are also sometimes identified.

The clinical manifestations of the disease can be diverse, owing to the hematogenous spread to virtually any organ site, the lungs being the most common organ involved, in up to $92 \%$ of patients ${ }^{4}$, which can result in frank respiratory failure requiring ventilatory support in $15.5 \%$ of cases $^{5}$.

Treatment recommendations (for which there is no evidence-based studies) involve antibiotic therapy with anaerobic coverage, surgical control of primary or secondary infectious sites and supportive therapy and duration of therapy has a reported median of 42 days, reflecting the different presentation, severity and course of the disease ${ }^{4}$. The role of anticoagulation is controversial, owing to the septic origin of thrombosis and fear of facilitating septic embolism and hemorrhagic complications².

\section{CASE REPORT}

A previously healthy 24-year-old female, smoker, overweight and with a history of depression, was admitted to our institution following the onset of sore throat, fever, nausea, vomiting and generalized malaise in the 4 days prior to admission. Two days before admission she had been started on IM penicillin $G$ and ibuprofen by her family physician, but her symptoms persisted and her malaise aggravated. Physical examination showed an erythematous oropharynx and a palpable lymphadenomegaly on the left side of her neck. The patient showed signs of dehydration, hypotension (80/40 $\mathrm{mmHg}$ ), tachycardia (100 bpm) and fever $\left(39^{\circ} \mathrm{C}\right)$. Inflamma-

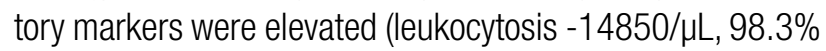
neutrophils-, C-reactive protein $327.6 \mathrm{mg} / \mathrm{L}$ ), she had acute kidney injury (urea $121 \mathrm{mg} / \mathrm{dL}$, creatinine $2.3 \mathrm{mg} / \mathrm{dL}$ ) and severe thrombocytopenia (15000/ $\mathrm{LL})$. Lactate was 1.9 $\mathrm{mmol} / \mathrm{L}$. A chest $X$-ray showed faint bilateral lower infiltrates and an abdominal and renal ultrasound excluded complications. She was started on iv ceftriaxone plus azithromycin and received fluid resuscitation. Despite treatment, over the first 24 hours acute respiratory failure impended and was admitted to the ICU where she was initially managed with non-invasive ventilation. A chest CT (figure 1) at this point showed bilateral irregular nodular opacities dispersed across the entire lung parenchyma suggestive of septic embolization. A gram positive diplococcus species grew in the blood cultures and group C beta-hemolytic Streptococcus was identified. There was no echocardiographic evidence 
Fig. 1
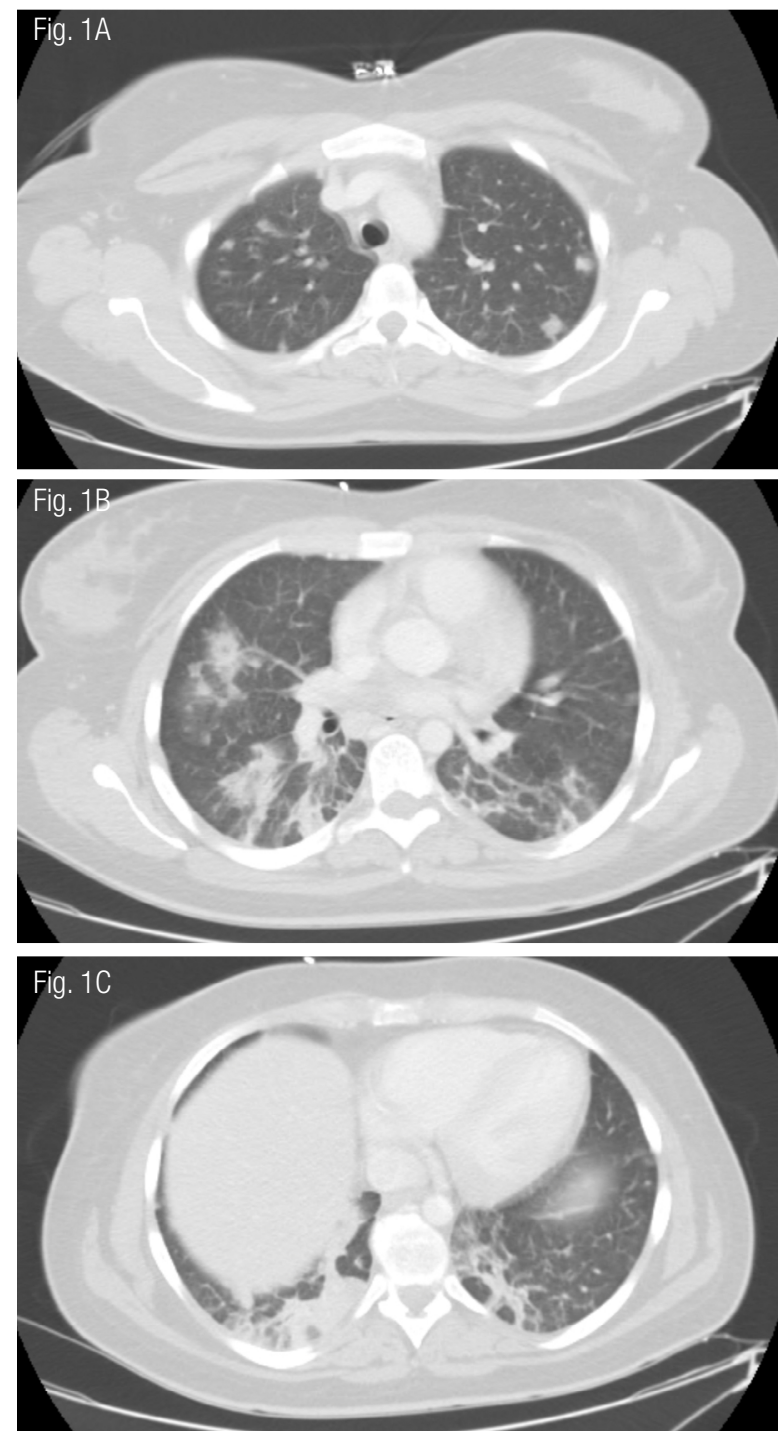

of endocarditis and antibiotic therapy was de-escalated to penicillin $\mathrm{G}$ according to the sensitivity chart. A neck CT (figure 2) showed partial venous thrombosis of the left internal jugular vein along much of its extension, a finding that was confirmed with a neck ultrasound, with accompanying extensive inflammatory edema of the left amygdaline region. A diagnosis of Lemierre's syndrome was established.

Over the next 72 hours, despite antibiotic therapy, the patient had further respiratory deterioration which motivated the start of invasive ventilation and escalation of antibiotic therapy to piperacillin/tazobactam. The deterioration was attributed to the development of acute respiratory distress syndrome (ARDS) and to the aggravation of the pulmonary lesions, which now showed extensive consolidation and necrotization of the lung parenchyma along with empyema. With insufficient response to a prone protocol, along with further difficulty ventilating the patient, at 7 days post ICU admission, the patient was transferred to a specialized center where she was placed under venovenous extracorporeal membrane oxygenation (ECMO). After over 6 weeks of anti-
Fig. 2
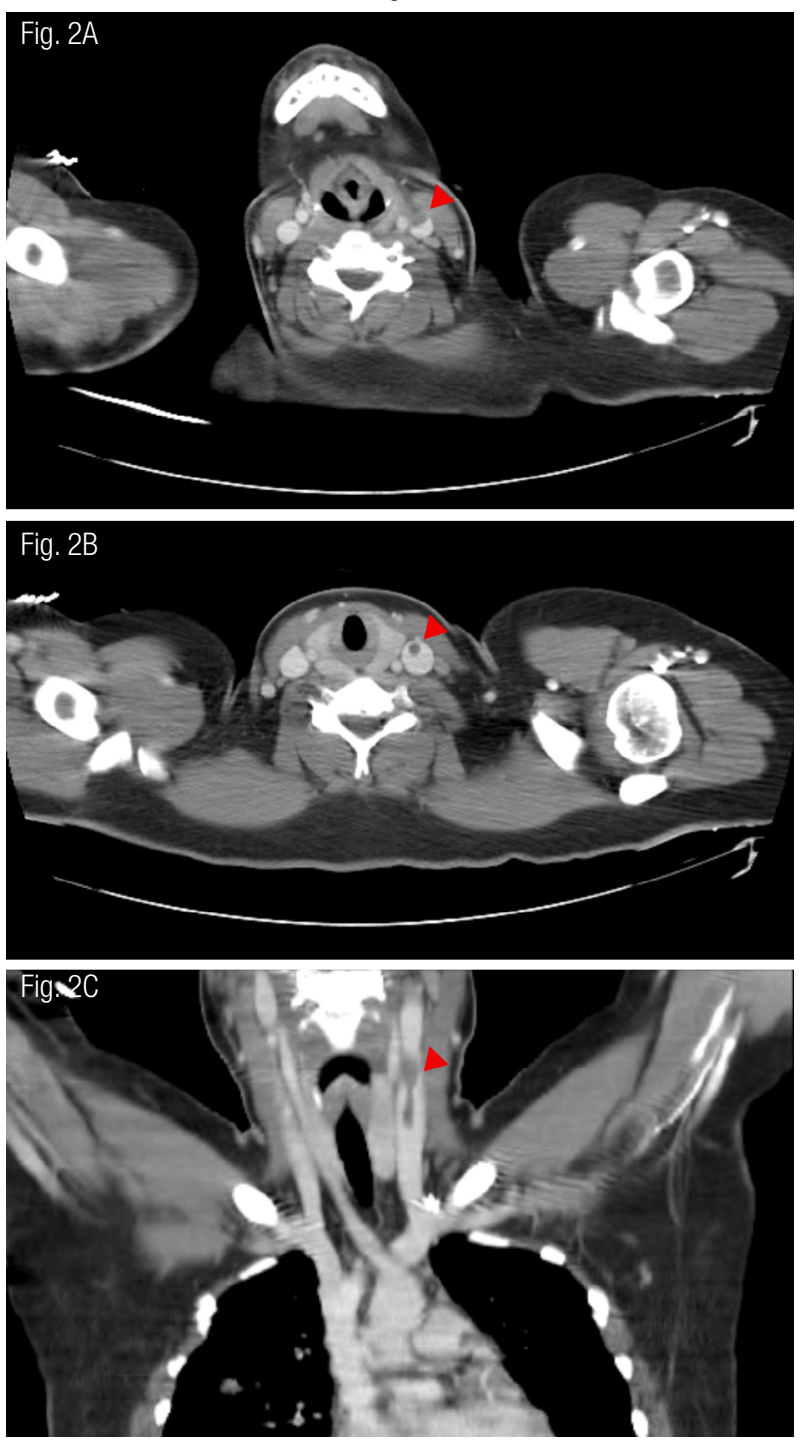

biotic therapy (later de-escalated to amoxicillin/clavulanate), over 30 days of ECMO and months of physical therapy after hospital discharge, she fully recovered and resumed a normal life.

\section{DISCUSSION}

The patient showed a triad of oropharyngeal infection, internal jugular vein thrombosis and septic lung lesions, that allowed a diagnosis of Lemierre's syndrome to be established early. Even though a Fusobacterium spp. was not isolated at any point, there are two relevant concepts to keep in mind. While anaerobic species cultures tend to be more technically challenging (often showing delayed growth as is the case of Fusobacterium spp.), in over 25\% of cases a mixed culture with some other bacterium species occurs ${ }^{4}$. It has also been previously described that group C Streptococcus and Fusobacterium necrophorum are often found together in patients with acute pharyngitis ${ }^{6}$ possibly representing a synergic pathogenic connection. This is especially relevant considering 4 cases of Lemierre's syndrome where co- 
infections with F. necrophorum and group C Streptococcus were also described ${ }^{4}$.

Similarly to a Lemierre's syndrome case published by Blossom et al. ${ }^{7}$, whose microbiological isolation was a group $C$ Streptococcus without evidence of Fusobacterium spp., we question whether the delayed growth of Fusobacterium spp. in the blood cultures was overlooked. Even though there were likely other factors at play when our patient continued to deteriorate even after appropriately guided antibiotic therapy with iv penicillin G for group C Streptococcus, lack of coverage for a potentially unidentified anaerobic species could be considered. While there is no evidence-based guidelines for treatment of Lemierre's syndrome, metronidazole seems to be the choice, mixing an excellent activity against Fusobacterium spp., as well as having good penetration into tissue ${ }^{4}$. Our patient's course was further complicated by a complex interplay of pulmonary dysfunction, which included lung abscesses, necrotizing pneumonia, empyema and ARDS $2,4,8$. While all of these have been previously reported, the quick onset of a degree of severity that led to an inability to adequately ventilate the patient and the definition of a need for support with ECMO, has only been described in a select number of cases with reported successful outcome ${ }^{9,10}$.

Regardless, prompt initiation of antibiotic therapy and early support of the patient in case of sepsis is the most important step in determining the outcome of the disease. Signs of septic embolization in a patient with a recent history of an oropharyngeal infection, should prompt the search thrombophlebitis and vice-versa, as well as the careful consideration for a potential delayed growth of an anaerobic species in microbiological cultures, even if another aerobic agent was already identified.

\section{REFERENCES}

1. Lemierre A. On certain seticaemias due to anaerobis organisms. Lancet. 1936:227:701-3.

2. Sacco C, Zane F, Granziera S, et al. Lemierre Syndrome: Clinical Update and Protocol for a Systematic Review and Individual Patient Data Meta-analysis. Hamostaseologie. 2019;39(1):76-86.

3. Hagelskjaer Kristensen L, Prag J. Lemierre's syndrome and other disseminated Fusobacterium necrophorum infections in Denmark: a prospective epidemiological and clinical survey. Eur J Clin Microbiol Infect Dis. 2008;27(9):779-789.

4. Riordan T. Human infection with Fusobacterium necrophorum (Necrobacillosis), with a focus on Lemierre's syndrome. Clin Microbiol Rev. 2007;20(4):622-659.

5. Chirinos JA, Lichtstein DM, Garcia J, Tamariz LJ. The evolution of Lemierre syndrome: report of 2 cases and review of the literature. Medicine (Baltimore). 2002:81(6):458-465.

6. Jensen A, Hagelskjaer Kristensen L, Prag J. Detection of Fusobacterium necrophorum subsp. funduliforme in tonsillitis in young adults by real-time PCR. Clin Microbiol Infect. 2007;13(7):695-701.

7. Blossom DB, Shah MD, Armitage KB. Lemierre Syndrome Caused by Group C Streptococcus. Inf Diseases in Clin Practice 2005;13:324-6.

8. Cosgrove EF, Colodny SM, Pesce RR. Adult respiratory distress syndrome as a complication of postanginal sepsis. Chest. 1993;103(5):1628-1629.

9. Blasco V, Leone M, Xeridat F, Albanèse J, Martin C. Syndrome de Lemierre avec pneumonie nécrosante assistée avec succès par épuration extracorporelle de $\mathrm{CO} 2$ [Lemierre's syndrome from necrotizing pneumonia treated with extracorporeal $\mathrm{CO} 2$ removal]. Ann Fr Anesth Reanim. 2008;27(3):244-248.

10. Carre AC, David JS, Mahr A, Flamens C, Fuster P, Bastien O. Utilisation de I'ECMO comme technique de sauvetage d'une hypoxémie réfractaire compliquant un syndrome de Lemierre [Use of ECMO as a salvage therapy for refractory hypoxia secondary to a Lemierre's syndrome]. Ann Fr Anesth Reanim. 2011;30(6):512515 